between regional brain activity and behaviour in psychiatric disorders (Gur et al, 1992). In my opinion 'affect' could also have been used as a probe to accomplish the objective of this study.

The rationale for considering affect as an appropriate probe in the evaluation of prefrontal activity comes from the literature that substantiate the role of frontal lobe in emotional behaviour (Akert, 1964; Robinson et al, 1984). Further, in view of the fact that psychopathology of emotional disturbance in schizophrenia and depression varies significantly, one may assume that these conditions may be associated with differential frontal lobe activity to emotional challenge, complementing the observed finding that showed abnormal regional cortical blood flow in frontal lobes following cognitive activation in schizophrenic patients compared with depressive patients.

The application of this strategy using emotional challenge would also allow us to observe the differential frontal activity to emotional and cognitive tasks in normals. But the non-availability of standardised emotional tasks that can be used as a probe limits the practical application of this strategy. However, valuable guidelines provided by Gur et al (1992) regarding conceptualisation, construction and standardisation of new neurobehavioural probes would be helpful in the development of the procedure and application of emotional challenge in future research.

AKERT, K. (1964) Comparative Anatomy of Frontal Cortex and Thalamo-Frontal Connections in the Frontal Granular Cortex and Behaviour (eds J. M. Warren \& K. Alert). New York: McGran McGraw-Hill.

Robinson, R. G., Kubos, K. L., Starr, L. B., et al (1984) Mood disorders in stroke patients: importance of location of lesion. Brain, 107, 81-93.

GUR, R. C., ERWIN, R. J. \& GUR, R. E. (1992) Neurobehavioural probes for psychologic neuroimaging studies. Archives of General Psychiatry. 49, 409-414.

Toronto Hospital

399 Bathurst Street

Toronto, Ontario

Canada M5T 2S8

R. RAMASUBBU

\section{Clinical studies of the dementias}

SIR: It was flattering to be so quoted by Dr Brayne (Journal, April 1993, 162, 439-446). While presenting an interesting epidemiological view of clinicopathological studies in dementia, some comments are necessary to put the issues in perspective for the general psychiatric reader. She is correct to highlight the importance of the sampling frame in such studies, but the sources of bias to which she refers pale into insignificance when one considers the proportion of people who come to post-mortem, an issue not addressed by the author. We achieved a positive response rate of about $75 \%$ which we believe to be reasonable (Burns et al, 1990a). Post-mortem rates of $100 \%$ would be misleading as people not consenting to the procedure would inevitably be excluded. To imagine that the epidemiological method should or could be uncritically applied to a procedure comparatively rarely undertaken seems incorrect. Also, in epidemiological studies the number of demented patients is in the minority, and so an enormous amount of work and normal post-mortems would be required to get a so-called 'representative sample' of those with dementia. As stated in the lively correspondence following the publication of our study, we would have sorely liked to have included more detail of the sampling frame, but could not due to lack of space.

The finding of particular clinical features in a sample does depend from where the sample is drawn and it is to be accepted that those samples with a psychiatric bias may include more with behavioural disturbance or psychiatric symptoms. The case that Lewy body dementia is peculiarly associated with such disturbances is unproven, as is Dr Brayne's assumption that this may have led to the relatively high proportion of Lewy body cases in our series - in fact, our series does not contain a larger number than others (Perry et al, 1989).

The introduction of clinical criteria for the dementias has a number of aims and should not be seen as the exclusive purview of the epidemiologist. To achieve an accurate diagnosis in an individual case is an important clinical objective. This was one of the main aims of our study and although we were rightly chastised for the use of the term 'sensitivity', the title of our paper 'Accuracy' should not have misled even the most naïve reader (Burns et al, 1990b). We do not agree that the reason for the different positive predictive values in our own study and that of Homer et al (1988) was due to a differing prevalence of Alzheimer's disease. Homer et al did not employ any recognised clinical criteria, indeed it was as a reaction to that paper and the associated widespread diagnostic nihilism which prompted us to report our own study in the same journal. Diagnostic criteria are being developed for vascular dementia. In fact, followers of that diagnostic group now have two to choose from! (Chiu et al, 1992; Roman et al, 1993.)

Dr Brayne is to be congratulated for producing such a timely review of this important issue. Clearly, dementia is such a hard nut to crack that contributions from a number of different perspectives are 
essential. We hope that our future work attracts similar attention-bad publicity is better than no publicity at all.

Burns, A., Reith, M., Levy, R., el al (1990a) "How to do it"obtaining consent for autopsy in Alzheimer's disease. International Journal of Geriatric Psychiatry, 5, 183-286.

- , LUTHERT, P., LEVY, R., et al (1990b) Accuracy of clinical diagnosis of Alzheimer's disease. British Medical Journal, 301 1026.

Chui, H. C., Victoroff, J. I., MARgolin, D., el al (1992) Criteria for the diagnosis of ischemic vascular dementia proposed by the State of California Alzheimer's Disease Diagnostic and Treatmen Centers. Neurology, 42, 473-480.

Homer, A., Honovar, M., Lantos, P., el al (1988) Diagnosing dementia: do we get it right? British Medical Journal, 297, 894-896.

Perry, R., Irving, D., Blessed, G., et al (1989) A clinically and neuropathologically distant form of dementia in the elderly. Lancet, $i, 166$.

Roman, G. C., Tatemichi, T. K. Erkinjunttr, T. et al (1993) Vascular dementia: diagnostic criteria for Research Studies Report of the NINDS-AIREN International Workshop. Neurology, 43, 250-260.

University of Manchester

Department of Old Age Psychiatry

Withington Hospital

Manchester M208LR

Institute of Psychiatry

RAYMOND LEVY

De Crespigny Park

Denmark Hill

London SE5 8AF

SIR: The article by Dr Brayne (Journal, April 1993, $162,439-446)$ concentrates on dementia in the general population but fails to discuss recent findings and recommendations as applied to the largest single group of people who develop Alzheimer's dementia; that is people with Down's syndrome. Difficulties inherent in the application of standard criteria (CAMDEX, DSM-III-R, NINCDS-ADRDA) in people with a learning disability have not been adequately investigated. Such definitions and criteria are difficult to apply to this population when falsepositive diagnoses may occur due to difficulty in testing (due to sensory handicap or loss, poor cooperation with testing), confounding illnesses being present (e.g. thyroid disorder), and also due to lack of knowledge of the ageing process in older people with Down's syndrome.

A greater emphasis can be placed on behavioural and neurological changes as these are often valuable in the diagnosis of dementia (Lai \& Williams, 1989; Evenhuis, 1990). Serial changes in behaviour over time in people with Down's syndrome may be used to support the clinical diagnosis of dementia (Prasher et al, 1993).

The relationship between clinical measurements and post-mortem findings must remain controversial as such findings may not necessarily correlate, as is the case with people with Down's syndrome. Virtually all people over the age of $\mathbf{4 0}$ years have neuropathological changes of Alzheimer's dementia, but not all will have changes of clinical dementia (Lai \& Williams, 1989).

EvenHuis, H. M. (1990) The natural history of dementia in Down's syndrome. Archives of Neurology, 47, 263-267.

LAI, F. \& WILLIAMS, R.S. (1989) A prospective study of Alzheimer's disease in Down's syndrome. Archives of Neurology, 46, 849-853.

Prasher, V. P., Krishinan, V. H. R., Clarke, D. J., et al (1993) The assessment of dementia in people with Down's syndrome; changes in adaptive behaviour. British Journal of Developmental Disability (in press).

V. Prasher

V. H. R. KRISHNAN

Queen Elizabeth Psychiatric Hospital

Mindelsohn Way

Birmingham B15 2QZ

\section{Lewy bodies and Alzheimer's disease}

SIR: Förstl et al (Journal, March 1993, 162, 385-392) have described the clinical characteristics of eight patients meeting NINCDS criteria for Alzheimer's disease but subsequently showing cortical Lewy bodies at autopsy. Although we agree that a signifcant number of patients meeting existing clinical diagnostic criteria for Alzheimer's disease will have Lewy body pathology, our own experience is that such patients are not representative of the clinical presentations more typically associated with cortical Lewy body disease.

Förstl et al report that the "Lewy body variant" patients in their sample were clinically no different from those without Lewy bodies, except for an increase in rigidity developing during the course of illness. This is surely an inevitable consequence of the case selection bias, only patients fulfilling NINCDS clinical criteria having been selected for study and those with other psychiatric presentations excluded. Our experience of $\mathbf{4 1}$ autopsy-confirmed cases of Lewy body dementia (McKeith et al, 1992a, 1992b) is that $80 \%$ present with a syndrome of fluctuating $\operatorname{cog}$ nitive impairment associated with hallucinations (usually visual), and with Parkinsonian features which are often precipitated by neuroleptics. A minority $(10-15 \%)$ of Lewy body patients lack this fluctuating picture and are therefore similar to those described by Förstl et al. A further 5-10\% present 